TRANSACTIONS OF THE

AMERICAN MATHEMATICAL SOCIETY

Volume 357, Number 3, Pages 1013-1023

S 0002-9947(04)03632-3

Article electronically published on October 5, 2004

\title{
THE NUMBER OF CERTAIN INTEGRAL POLYNOMIALS AND NONRECURSIVE SETS OF INTEGERS, PART 2
}

\author{
HARVEY M. FRIEDMAN
}

\begin{abstract}
We present some examples of mathematically natural nonrecursive sets of integers and relations on integers by combining results from Part 1 , from recursion theory, and from the negative solution to Hilbert's 10th Problem.
\end{abstract}

\section{INTRODUCTION}

We present some examples of mathematically natural nonrecursive sets of integers and multivariate relations on integers.

The usual examples of nonrecursive sets and relations on the integers involve mathematically unnatural codings of finite objects as integers, and may also involve models of computation and formal languages.

A very important mathematically natural example of a nonrecursive set of finite objects comes from the work on Hilbert's 10th Problem (see [2]). This is the set of all integral polynomials that have an integral zero. This example avoids models of computation and formal languages. However, it does not readily provide a mathematically natural nonrecursive set of integers. For example, one can form the nonrecursive set of integer codes of integral polynomials that have an integral zero. However, the coding of integral polynomials as integers destroys the mathematical naturalness of the example.

Our approach is to use the work on Hilbert's 10th Problem together with [3]. In particular, we show that the following two sets are nonrecursive (see Corollary 26).

$\left\{n \in Z^{+}:(\exists P \in I P O L Y)\left(n=\max (P Z)\right.\right.$ and $\left.\left.P[-3,3] \subseteq\left[-(\log n)^{1 / 3},(\log n)^{1 / 3}\right]\right)\right\} ;$

$\left\{n \in Z^{+}:(\exists P \in I P O L Y)\left(n=\left|P Z \cap Z^{+}\right|\right.\right.$and $\left.\left.P[-3,3] \subseteq\left[-(\log n)^{1 / 3},(\log n)^{1 / 3}\right]\right)\right\}$.

We also show that if $-3,3$ are replaced by $-3 / 2,3 / 2$, then both of these sets become recursive. See Corollary 26

Here IPOLY is the set of all integral polynomials (polynomials of several variables with integer coefficients into $\Re$ ). For $E \subseteq \Re, P E$ is the set of all values of $P$ at arguments from $E$. Here || is used for cardinality. log is the natural logarithm.

Some readers may be uncomfortable with the informal notion of "mathematically natural" in this context. We can restate the aim as one of finding examples that are "more and more mathematically natural". Readers who are still uncomfortable will find rich connections between analysis, recursion theory, and Hilbert's 10th problem that are of interest in their own right.

Received by the editors July 15, 2003.

2000 Mathematics Subject Classification. Primary 03D20, 03D80; Secondary 11U05.

(C)2004 American Mathematical Society 


\section{Some RECURSION THEORETIC SETS}

We use $N$ for the set of all nonnegative integers. In this section, we will use $W_{e}$ for the r.e. enumeration of the r.e. sets given by a standard Turing machine model, and $\varphi_{e}(n)$ for a standard partial recursive enumeration of the partial recursive functions, also given by a standard Turing machine model. In section 3, we will work with any standard enumeration.

Let $A=\left\{n \in N:(\exists e<n)\left(n=\max \left(W_{e}\right)\right)\right\}$. Let $B=\{n \in N:(\exists e<n)(n=$ $\left.\left.\left|W_{e}\right|\right)\right\}$. We will show that $A, B$ are not r.e. and not co-r.e.

First we need an easy combinatorial lemma.

Lemma 1. Let $f: N \rightarrow N$, where no tail of $f$ is one-one. The set $X=\{n \in N$ : $(\exists e<n)(n=f(e))\}$ is coinfinite.

Proof. Let $p \in N$. Choose $q>p$ such that the range of $f$ on $[p, q]$ has at most $|[p, q]|-p-1$ elements. In particular, $f$ on $[p, q]$ omits at least $p+1$ elements of $[p, q]$. Hence $f$ on $[0, q]$ omits some $n \in[p, q]$. Therefore $n \notin X$.

Lemma 2. A, B are not r.e.

Proof. Suppose $A$ is r.e., and let $A=W_{e}$, where $e$ is sufficiently large. By Lemma 1 let $r$ be largest such that $\left[2^{e}, r\right) \subseteq A$. Then $r \notin A$. Note that $\left[2^{e}, r\right]=\left\{i \geq 2^{e}\right.$ : $\left.\left[2^{e}, i\right) \subseteq A\right\}$ is $W_{e^{\prime}}$ for some $e^{\prime}<2^{e}$, and nonempty. Hence $\max \left(\left[2^{e}, r\right]\right)=r \in A$.

Suppose $B$ is r.e., and let $B=W_{e}$, where $e$ is sufficiently large. By Lemma 1 let $r$ be largest such that $\left[2^{e}, r\right) \subseteq B$. Then $r \notin B$. Note that $\left[2^{e}, 2^{e}+r\right)$ is $W_{e^{\prime}}$ for some $e^{\prime}<2^{e}$. Hence $\left|\left[2^{e}, 2^{e}+r\right)\right|=r \in B$.

Theorem 3. $A$ is not r.e. and not co-r.e. A meets every infinite r.e. set. $A$ is recursive in $0^{\prime}$.

Proof. For the second claim, let $A$ be disjoint from the infinite r.e. set $W_{e}$. We can assume that $e$ is sufficiently large. Let $p$ be the first element greater than $2^{e}$ that comes up in the enumeration of $W_{e}$. Then $\{p\}=W_{e^{\prime}}$ for some $e^{\prime}<p$. Hence $p \in A$. This is a contradiction. By Lemma 1 is coinfinite. Hence $A$ is not co-r.e. $A$ is not r.e. by Lemma 2 To see that $A$ is recursive in $0^{\prime}$, note that $n \in A$ if and only if $(\exists e<n)\left(n \in W_{e}\right.$ and $\left.\left(\forall m \in W_{e}\right)(m \leq n)\right)$. The inside of this formula is the conjunction of a $\Sigma$ formula and a $\Pi$ formula, which is recursive in $0^{\prime}$. The outermost bounded quantifier will keep this formula recursive in $0^{\prime}$.

Theorem 4. $B$ is not r.e. and not co-r.e. $B$ meets every infinite r.e. set. $B$ is recursive in $0^{\prime}$.

Proof. For the second claim, let $B$ be disjoint from the infinite r.e. set $W_{e}$. We can assume that $e$ is sufficiently large. Let $p$ be the first element greater than $2^{e}$ that comes up in the enumeration of $W_{e}$. Then $\{1,2, \ldots, p\}=W_{e^{\prime}}$ for some $e^{\prime}<p$. Hence $p \in B$. This is a contradiction. By Lemma 1, $B$ is coinfinite. Hence $A$ is not co-r.e. $B$ is not r.e. by Lemma 2 To see that $B$ is recursive in $0^{\prime}$, note that $n \in B$ if and only if $(\exists e<n)$ (there are at least $n$ elements of $W_{e}$ and at most $n$ elements of $W_{e}$ ). The inside of this formula is the conjunction of a $\Sigma$ formula and a $\Pi$ formula, which is recursive in $0^{\prime}$. The outermost bounded quantifier will keep this formula recursive in $0^{\prime}$.

The set $A^{*}=\left\{n:(\exists e<n)\left(\varphi_{e}(0)=n\right)\right\}$ has been well studied. 
$E \subseteq N$ is called effectively simple if and only if

(1) $E$ is r.e.;

(2) $E$ is coinfinite; and

(3) there is a recursive function $g: N \rightarrow N$ such that for all $e$, if $W_{e}$ is disjoint from $E$, then $\left|W_{e}\right| \leq g(e)$.

Lemma 5. $A^{*}$ is effectively simple.

Proof. $A^{*}$ is obviously r.e., and by Lemma 1, $A^{*}$ is coinfinite. For each $e$, let $h(e)$ be the maximum element among the first $2^{e}$ elements of $W_{e}$ in the standard enumeration of $W_{e}$. Here $h(e)$ is defined if and only if there are at least $2^{e}$ elements of $W_{e}$. Then for sufficiently large $e$, if $h(e)$ is defined then $h(e) \geq 2^{e}$ and $h(e) \in A^{*}$. Hence for sufficiently large $e$, if $W_{e}$ is disjoint from $A^{*}$ then $h(e)$ is undefined. Therefore for sufficiently large $e$, if $W_{e}$ is disjoint from $A^{*}$ then $\left|W_{e}\right|<2^{e}$. Also, as in the proof of Theorem 3 for all $e$, if $W_{e}$ is disjoint from $A^{*}$ then $W_{e}$ is finite. So we can set $g$ to be $2^{e}$ for sufficiently large $e$, and sufficiently large for small $e$.

Lemma 6. Every effectively simple set is of Turing degree $0^{\prime}$.

Proof. For a proof of this theorem of D.A. Martin (as well as the above Lemma 5), see [1], pp. 263-265.

Theorem 7. $A^{*}$ is of Turing degree $0^{\prime}$.

Proof. By Lemmas 5 and 6

Note that we have relied on the assumption that we are working with $W_{e}$ and $\varphi_{e}$ based on a Turing machine model. In the next section, we prove much more general versions of these results which avoid such assumptions.

\section{SOME STRONG GENERALIZATIONS}

Here we replace the relation $n=\max \left(W_{e}\right)$ with the general relation $n=f(e)$. We also generalize $e<n$. We assume that $\varphi_{n}(m)$ is any standard partial recursive enumeration of the partial recursive functions (not necessarily given by a Turing machine model). See [1], p. 215.

Lemma 8. Let $g: N \rightarrow N$ be recursive. For all $r \geq 0$ there exist $n_{1}, \ldots, n_{r}$ such that $\varphi_{n_{1}}(0), \ldots, \varphi_{n_{r}}(0)$ are distinct and $>2 \max \left(g\left(n_{1}\right), \ldots, g\left(n_{r}\right)\right)$.

Proof. Let $g$ be as given. We prove the following by induction on $r \geq 0$. For all $t$, there exist $n_{1}, \ldots, n_{r}$ such that

$$
\varphi_{n_{1}}(0), \ldots, \varphi_{n_{r}}(0) \text { are distinct and }>2 \max \left(g\left(n_{1}\right), \ldots, g\left(n_{r}\right), t\right) .
$$

The basis case $r=0$ is vacuous. Assume that our assertion is true for a fixed $r \geq 0$. Let $t$ be given. By the recursion theorem, define

$$
\varphi_{n_{r+1}}(0) \cong 2 \max \left(g\left(n_{1}\right), \ldots, g\left(n_{r}\right), \varphi_{n_{1}}(0), \ldots, \varphi_{n_{r}}(0), g\left(n_{r+1}\right)\right)+1,
$$

where $n_{1}, \ldots, n_{r}$ are chosen by effective search such that $\varphi_{n_{1}}(0), \ldots, \varphi_{n_{r}}(0)$ are distinct and $>2 \max \left(g\left(n_{1}\right), \ldots, g\left(n_{r+1}\right), t\right)$. By the induction hypothesis, this effective search will be successful, and so

$$
\varphi_{n_{r+1}}(0)=2 \max \left(g\left(n_{1}\right), \ldots, g\left(n_{r}\right), \varphi_{n_{1}}(0), \ldots, \varphi_{n_{r}}(0), g\left(n_{r+1}\right)\right)+1 .
$$


Hence $\varphi_{n_{r+1}}(0)$ is distinct from $\varphi_{n_{1}}(0), \ldots, \varphi_{n_{r}}(0)$, and the latter are distinct and $>2 \max \left(g\left(n_{1}\right), \ldots, g\left(n_{r+1}\right), t\right)$. Also $\varphi_{n_{r+1}}(0)>2 \max \left(g\left(n_{1}\right), \ldots, g\left(n_{r+1}\right), t\right)$. This completes the induction argument.

Lemma 9. Assume

(1) $f: E \rightarrow N, E \subseteq N$; and

(2) there is a recursive function $g: N \rightarrow N$ such that for all $n$, if $\varphi_{n}(0)$ exists then $\varphi_{n}(0)=f(g(n))$.

Then for all $r \geq 1$ there exists $n_{1}<\ldots<n_{r}$ such that $f\left(n_{1}\right), \ldots, f\left(n_{r}\right)>2 n_{r}$.

Proof. Let $f, E, g$ be as given. By Lemma 8 , let $m_{1}, \ldots, m_{r}$ be such that $\varphi_{m_{1}}(0), \ldots$, $\varphi_{m_{r}}(0)$ are distinct and greater than $\max \left(g\left(m_{1}\right), \ldots, g\left(m_{r}\right)\right)$. Then $f\left(g\left(m_{1}\right)\right), \ldots$, $f\left(g\left(m_{r}\right)\right)$ are distinct and $>2 \max \left(g\left(m_{1}\right), \ldots, g\left(m_{r}\right)\right)$. Choose $n_{1}, \ldots, n_{r}$ to be $g\left(m_{1}\right)$, ..., $g\left(m_{r}\right)$ in increasing order.

Theorem 10. Assume

(1) $f: E \rightarrow N, E \subseteq N$;

(2) there is a recursive function $g: N \rightarrow N$ such that for all $n$, if $\varphi_{n}(0)$ exists then $\varphi_{n}(0)=f(g(n))$; and

(3) $h: N \rightarrow N$ is recursive, unbounded, and $h(n)-n$ is bounded above.

Then the set $S[f, h]=\{n: n \in f[0,1, \ldots, h(n)]\}$ meets every infinite r.e. set and is coinfinite. In particular, $S[f, h]$ is not co-r.e.

Proof. Let $s \geq 1$ be an upper bound for $h(n)-n$. Hence $h(n) \leq n+s$. To see that $S[f, h]$ is coinfinite, it suffices to show that $S^{\prime}=\{n: n \in f[0,1, \ldots, n+s]\}$ is coinfinite. Let $t \geq 1$. We show that $S^{\prime}$ excludes some $m \geq t$. Let $r \gg s, t$, and $n_{1}<\ldots<n_{r}$, where $f\left(n_{1}\right), \ldots, f\left(n_{r}\right)>2 n_{r}$, using Lemma 9 Note that $f\left[0, \ldots, n_{r}+s\right]$ has $n_{r}+s+1$ values, counting multiplicities, and at least $r$ of these values lie above $n_{r}+s$. Hence $f\left[0, \ldots, n_{r}+s\right]$ has at most $n_{r}+s+1-r$ distinct values in $\left[0, \ldots, n_{r}+s\right]$. Therefore at least $r$ elements of $\left[0, \ldots, n_{r}+s\right]$ must lie outside $f\left[0, \ldots, n_{r}+s\right]$. Hence at least $t+1$ elements of $\left[0, \ldots, n_{r}\right]$ lie outside $f\left[0, \ldots, n_{r}+s\right]$. These $t+1$ elements cannot lie in $S^{\prime}$. Hence $S^{\prime}$ excludes some $m \geq t$.

It suffices to show that $S[f, h]$ meets every infinite recursive set. Let $E$ be an infinite recursive set. By the recursion theorem and the fact that $h$ is unbounded, define $\varphi_{n}(0)$ to be the least element $p \in E$ such that $h(p)>g(n)$. Then $\varphi_{n}(0)=$ $p=f(g(n))$ and $h(f(g(n)))>g(n)$. We claim that $f(g(n))=\varphi_{n}(0) \in S[f, h]$. This follows from $g(n) \in[0,1, \ldots, h(f(g(n)))]$.

Theorem 11. Assume

(1) $f: N \rightarrow N$ is partial recursive;

(2) there is a recursive function $g: N \rightarrow N$ such that for all $n$, if $\varphi_{n}(0)$ exists then $\varphi_{n}(0)=f(g(n)) ;$ and

(3) $h: N \rightarrow N$ is recursive, unbounded, and $h(n)-n$ is bounded above.

Then the set $S[f, h]=\{n: n \in f[0,1, \ldots, h(n)]\}$ is effectively simple, and hence is of Turing degree $0^{\prime}$.

Proof. By Theorem [10, $S[f, h]$ is coinfinite. Also $S[f, h]$ is obviously r.e. Let $W_{e}$ be disjoint from $S[f, h]$. Let $x_{1}, x_{2}, \ldots$ be the standard effective listing of the elements of $W_{e}$ without repetition. The listing may not be in increasing order. We wish to obtain an effective upper bound on the length of this listing in terms of $e$. 
By the recursion theorem, let $n=n(e)$ be such that $\varphi_{n}(0)$ is the first element $p$ in the enumeration of $W_{e}$ such that $h(p)>g(n)$. Here $\varphi_{n}(0)$ converges if and only if $p$ exists. Suppose $n=n(e)$ exists. Then $h(f(g(n)))>g(n)$. We claim that $f(g(n)) \in S[f, h]$. This follows from $g(n) \in\{0,1, \ldots, h(f(g(n)))\}$. This is a contradiction. We have now shown that for all $e$, if $W_{e}$ is disjoint from $S[f, h]$, then $n(e)$ does not exist. Let $G(e)$ be the number of $p$ such that $h(p) \leq g(n)$. Then in the enumeration of $W_{e}$, at most $G(e)+1$ integers can appear. Hence $\left|W_{e}\right| \leq G(e)+1$.

We say that $h: N \rightarrow N$ goes to $\infty$ if and only if for all $n \geq 1, h$ is eventually greater than $n$.

Theorem 12. Let $h: N \rightarrow N$ be recursive and go to $\infty$, and let $h(n)-n$ be bounded above. Then the sets

$$
\text { 1) }\left\{n \in N:(\exists e \leq h(n))\left(n=\max \left(W_{e}\right)\right)\right\} \text {, }
$$$$
\text { 2) }\left\{n \in N:(\exists e \leq h(n))\left(n=\left|W_{e}\right|\right)\right\}
$$

meet every infinite r.e. set, and are not r.e. and not co-r.e. On the other hand, let $h: N \rightarrow N$ be recursive, eventually strictly increasing, and omit infinitely many values. There are standard enumerations $W_{e}, \varphi_{n}$, such that the sets

3) $\left\{n \in N:(\exists e \leq h(n))\left(n=\max \left(W_{e}\right)\right)\right\}$,

4) $\left\{n \in N:(\exists e \leq h(n))\left(n=\left|W_{e}\right|\right)\right\}$,

5) $\left\{n \in N:(\exists e \leq h(n))\left(n=\varphi_{e}(0)\right)\right\}$

are all cofinite.

Proof. By Theorem 10, 1) and 2) meet every infinite r.e. set, are coinfinite, and hence are not co-r.e. For the last claim about cofiniteness, note that $N \backslash(\operatorname{rng}(h)-1)$ is an infinite recursive set. We can arrange for a standard enumeration to focus its action on indices in that infinite recursive set, whereas we arrange that for all positive $h(n), \varphi_{h(n)-1}(0)=n$. Under this standard enumeration, 5$)$ is cofinite. If we arrange that for all positive $h(n), W_{h(n)-1}=\{1, \ldots, n\}$, then 3) and 4) are cofinite.

To see that 1) and 2) are not r.e., we adapt the proof of Lemma 2 to work for any standard enumeration. Suppose 1) is r.e. By the recursion theorem, define $W_{e}$ to consist of those $n$ for which $h(n) \geq e$, where all $n^{\prime}<n$ with $h\left(n^{\prime}\right) \geq e$ lie in 1 ). If $W_{e}$ is infinite, then 1) contains all $n$ for which $h(n) \geq e$, which contradicts that 1 ) is coinfinite (Theorem 10). Hence $W_{e}$ is finite and obviously nonempty. Also by the definition of $W_{e}$, we see that $\max \left(W_{e}\right)$ does not lie in 1$)$. However, $h\left(\max \left(W_{e}\right)\right) \geq e$, and $\max \left(W_{e}\right)$ lies in 1$)$.

Suppose 2) is r.e. By the recursion theorem, define $W_{e}$ to consist of those $i>0$ for which there exists an $n \geq i$ for which $h(n) \geq e$, where all $n^{\prime}<n$ with $h\left(n^{\prime}\right) \geq e$ lie in 2). If $W_{e}$ is infinite, then 2) contains all $n$ for which $h(n) \geq e$, which contradicts that 2 ) is coinfinite (Theorem 10). Hence $W_{e}$ is finite and obviously nonempty. Also by the definition of $W_{e}$, we see that $\max \left(W_{e}\right)=\left|W_{e}\right|$ does not lie in 2). However, $h\left(\left|W_{e}\right|\right) \geq e$, and $\left|W_{e}\right|$ lies in 2).

\section{NATURAL NONRECURSIVE SETS}

By an integral polynomial we mean a polynomial function of the form $P: \Re^{k} \rightarrow$ $\Re$ presented as a polynomial with integer coefficients. Let IPOLY be the set of 
all integral polynomials. We can treat IPOLY recursion-theoretically in terms of codes for the presentations of integral polynomials.

Lemma 13. Assume

(1) $P_{0}, P_{1}, \ldots$ is a recursive enumeration of IPOLY without repetition;

(2) $f: E \rightarrow N, E \subseteq I P O L Y$;

(3) there is a recursive function $g: N \rightarrow I P O L Y$ such that for all $n$, if $\varphi_{n}(0)$ exists then $\varphi_{n}(0)=f(g(n))$; and

(4) $h: N \rightarrow N$ is recursive and unbounded, while $h(n)-n$ is bounded above.

Then $\left\{n: n \in f\left[P_{0}, P_{1}, \ldots, P_{h(n)}\right]\right\}$ meets every infinite r.e. set and is coinfinite. In particular, it is not co-r.e.

Proof. Let the P's, $f, g, h$ be as given. Let $f^{\prime}: N \rightarrow N$ be given by $f^{\prime}(n)=f\left(P_{n}\right)$. Let $g^{\prime}: N \rightarrow N$ be given by $g^{\prime}(n)=m$, where $g(n)=P_{m}$. Then $g^{\prime}$ is recursive. Also if $\varphi_{n}(0)$ exists then $\varphi_{n}(0)=f(g(n))=f\left(P_{g^{\prime}(n)}\right)=f^{\prime}\left(g^{\prime}(n)\right)$. So $f^{\prime}, g^{\prime}, h$ obey the hypotheses of Theorem [10] To complete the proof, note that $\{n: n \in$ $\left.f^{\prime}[0, \ldots, h(n)]\right\}=\left\{n: n \in f\left[P_{0}, \ldots, P_{h(n)}\right]\right\}$.

For $A \subseteq \Re$, it is convenient to write $P A$ for the set of all values of $P$ at arguments from $A$. In this way, we can avoid introducing a letter for the number of variables of $P$.

Let $A$ be a nonempty compact subset of $\Re$. We write $\operatorname{maxmag}(A)$ for the maximum of the absolute values of the elements of $A$.

Lemma 14. Let $r>2$ and $n$ be sufficiently large. There are at most $n / 2 P \in$ $I P O L Y$ such that $P$ maps $[-r, r]$ into $\left[-(\log n)^{1 / 3},(\log n)^{1 / 3}\right]$. Furthermore, there is a recursive function $f(r, n)$ such that for all rationals $r>2$ and all integers $n \geq$ 0 , every $P \in I P O L Y$ which maps [-r, $r]$ into [-n,n] has number of variables, degree, and magnitude of coefficients bounded above by the integer $f(r, n)$.

Proof. For the first claim, see 3, Theorem 2. For the second claim, see the estimates in [3], Lemmas 1, 2, 3 .

Lemma 15. Let $r>2$ be rational. There is a recursive enumeration $P_{0}^{r}, P_{1}^{r}, \ldots$ of IPOLY without repetition, where for all $n$,

$$
\operatorname{maxmag}\left(P_{n}^{r}[-r, r]\right) \leq \operatorname{maxmag}\left(P_{n+1}^{r}[-r, r]\right) .
$$

Proof. Fix $r$ as given. We first claim that for each $n \geq 0$, we can effectively list the finitely many $P \in I P O L Y$ in increasing $(\leq)$ order of maxmag $(P[-r, r])$, where $\operatorname{maxmag}(P[-r, r]) \leq n$, without repetition. To see this, given $n$, first effectively list all $P \in I P O L Y$ whose number of variables, degree, and magnitude of coefficients are bounded above by $f(r, n)$, as in Lemma 14, without repetition. Call this the $n$-th pre-list. Using the Tarski decision procedure for the elementary theory of the field of the real numbers, we can give the presentation of any maxmag $(P[-r, r])$ as a real algebraic number, effectively in $P \in I P O L Y$, and then effectively compare any two such presentations, effectively in $P \in I P O L Y$. Hence by deleting terms and rearranging the order of terms as necessary, we can effectively convert the $n$-th pre-list into the $n$-th list as claimed. Now note that for all $n \geq 0$, the $n$-th list is an initial segment of the next list. We can now create the required recursive enumeration by combining these finite lists. 
The fundamental recursion-theoretic fact that we need about integral polynomials comes from the Matiyasevich/Robinson/Davis/Putnam solution to Hilbert's 10th problem (see [2]).

Lemma 16. Let $k \geq 1$ and $W \subseteq Z^{k}$ be r.e. There exist $m \geq 1$ and $P \in I P O L Y$ with $k+m$ variables such that $\bar{W}=\left\{x \in Z^{k}:\left(\exists y \in Z^{m}\right)(P(x, y)=0)\right\}$. Furthermore, every such set is r.e.

Proof. See [2]. We choose to have the existential quantifiers range over $Z$, and this reduces to the case where they range over $N$, because every nonnegative integer is the sum of four squares.

Lemma 17. There is a recursive function $T: N \rightarrow I P O L Y$ such that for all $e$, $T(e) Z \cap N=W_{e}$. There is a recursive function $J: N \rightarrow I P O L Y$ such that for all $n$, if $\varphi_{n}(0)$ exists then $J(n) Z \cap N=\left\{1, \ldots, \varphi_{n}(0)\right\}$.

Proof. The second claim follows immediately from the first. By Lemma 16 let $m \geq 1$ and $P \in I P O L Y$ with $2+m$ variables such that for all $e, n \in Z, n \in W_{e}$ if and only if $\left(\exists y \in Z^{m}\right)(P(e, n, y)=0)$. Define $T(e)$ to be the integral polynomial $Q(n, y)=n-\left(n^{2}+1\right) P(e, n, y)^{2}$. Note that if $P(e, n, y) \neq 0$, then $Q(n, y) \leq$ $n-\left(n^{2}+1\right)=n-n^{2}-1<0$. Therefore $Q(n, y) \geq 0$ implies $P(e, n, y)=0$ and $Q(n, y)=n$ and $n \in W_{e}$. Hence every nonnegative value of $Q(n, y)$ lies in $W_{e}$. On the other hand, if $n \in W_{e}$, then there exists $y \in Z^{m}$ such that $P(e, n, y)=0$ and $Q(n, y)=n$. So the nonnegative values of $Q$ are exactly the elements of $W_{e}$.

The construction used in the preceding proof was introduced in [4] to prove that every Diophantine set of positive integers is the positive range of an integral polynomial.

We define the following two sets for all real numbers $r$.

$$
\begin{gathered}
C[r]=\left\{n \in Z^{+}:(\exists P \in I P O L Y)(n=\max (P Z) \text { and }\right. \\
\left.\left.P[-r, r] \subseteq\left[-(\log n)^{1 / 3},(\log n)^{1 / 3}\right]\right)\right\}, \\
D[r]=\left\{n \in Z^{+}:(\exists P \in I P O L Y)\left(n=\left|P Z \cap Z^{+}\right|\right. \text {and }\right. \\
\left.\left.P[-r, r] \subseteq\left[-(\log n)^{1 / 3},(\log n)^{1 / 3}\right]\right)\right\} .
\end{gathered}
$$

Lemma 18. Let $r>2$ be rational. Then $C[r]$ and $D[r]$ each meet every infinite r.e. set, and are coinfinite. Also $C[r]$ and $D[r]$ are of Turing degree $\leq 0^{\prime}$.

Proof. Let $r$ be as given. We apply Lemma 13. We use the recursive enumeration of IPOLY in Lemma 15 Take $E=\{P \in I P O L Y: P Z$ is bounded above $\}$. Take $g=J$ of Lemma 17. Take $f_{1}(P)=\max (P Z)$ and $f_{2}(P)=\left|P Z \cap Z^{+}\right|$. By Lemma 14 let $t$ be so large that for all $n \geq t$, there are at most $n / 2 P \in I P O L Y$ such that $P$ maps $[-r, r]$ into $\left[-(\log n)^{1 / 3},(\log n)^{1 / 3}\right]$. Note that for $n>1,(\log n)^{1 / 3}$ is transcendental. Therefore we can effectively compare, for each $P \in I P O L Y$, the quantity maxmag $(P[-r, r])$ with $(\log n)^{1 / 3}$. This is because we know from the Tarski decision procedure for the field of real numbers that maxmag $(P[-r, r])$ is algebraic, and its presentation is given effectively from $P$. This means that we are comparing a given real algebraic number with an effectively given transcendental number. So for $n \geq t$, we define $h(n)$ as the greatest $m$ such that $\operatorname{maxmag}\left(P_{m}[-r, r]\right)<(\log n)^{1 / 3}$, using Lemma 14. For $n<t$, we define $h(n)=0$. Then for $n \geq t$ we have $h(n) \leq n / 2<n$, and $h$ is recursive and unbounded. Also, 
using $g=J$ from Lemma [17 we have established all of the hypotheses of Lemma 13 for $f_{1}, f_{2}$. By Lemma 13 we see that $C[r]$ and $D[r]$ each meet every infinite r.e. set, and are coinfinite.

For the last claim, we have only to effectively decide for any given $n>1$ whether or not $\left.P[-r, r] \subseteq[-\log n)^{1 / 3},(\log n)^{1 / 3}\right]$. We again use the Tarski decision procedure for the field of real numbers to effectively and explicitly obtain $\operatorname{maxmag}(P[-r, r])$ as a real algebraic number, and compare this to $(\log n)^{1 / 3}$, the latter being an effectively given transcendental.

Let $F, G: Z^{+} \rightarrow \Re^{+}$. We now define the following two sets.

$C[r, F]=\left\{n \in Z^{+}:(\exists P \in I P O L Y)(n=\max (P Z)\right.$ and $\left.P[-r, r] \subseteq[-F(n), F(n)])\right\}$,

$D[r, F]=\left\{n \in Z^{+}:(\exists P \in I P O L Y)\left(n=\left|P Z \cap Z^{+}\right|\right.\right.$and $\left.\left.P[-r, r] \subseteq[-F(n), F(n)]\right)\right\}$.

Lemma 19. Let $r>2$ be rational. Suppose $F: Z^{+} \rightarrow \Re^{+}$eventually dominates an unbounded recursive function from $Z^{+}$into $Z^{+}$, and for all sufficiently large $n$, $F(n) \leq(\log n)^{1 / 3}$. Then $C[r, F]$ and $D[r, F]$ meet every infinite r.e. set and are coinfinite.

Proof. We again apply Lemma 13, Take $E=\{P \in I P O L Y: P Z$ is bounded above $\}$. Take $g=J$ of Lemma 17 Take $f_{1}(P)=\max (P Z)$ and $f_{2}(P)=\left|P Z \cap Z^{+}\right|$. Let $F$ eventually dominate the unbounded recursive function $h: Z^{+} \rightarrow Z^{+}$. Let $n$ be sufficiently large, according to the hypotheses and Lemma 14 Since $F(n) \leq$ $(\log n)^{1 / 3}$, we see that $h(n) \leq n / 2$. We now apply Lemma 13] with $f_{1}$ and $f_{2}$, obtaining two sets $C, D$. Obviously all but finitely many elements of $C$ lie in $C[r, F]$, and all but finitely many elements of $D$ lie in $D[r, F]$. Since $C, D$ meet every infinite r.e. set, we see that $C[r, F]$ and $D[r, F]$ also meet every infinite r.e. set. On the other hand, by the inequality on $F$, we see that all but finitely many elements of $C[r, F]$ lie in $C[r]$, and all but finitely many elements of $D[r, F]$ lie in $D[r]$. Since the latter are coinfinite, so are the former.

Lemma 20. Let $r>2$ be a real number. Suppose $F: Z^{+} \rightarrow \Re^{+}$eventually dominates an unbounded recursive function from $Z^{+}$into $Z^{+}$, and for all sufficiently large $n, F(n) \leq(\log n)^{1 / 3}$. Then $C[r, F]$ and $D[r, F]$ meet every infinite r.e. set and are coinfinite. $C[r, F]$ and $D[r, F]$ are not co-r.e.

Proof. Let $r, F$ be as given. Let $2<p<r<q$, where $p, q$ are rationals. By Lemma $19] C[p, F], D[p, F], C[q, F], D[q, F]$ each meet every infinite r.e. set and are coinfinite. Hence by the obvious inclusion relations, this is also true of $C[r, F], D[r, F]$. It follows immediately that $C[r, F]$ and $D[r, F]$ are not co-r.e.

Lemma 21. Under the hypotheses of Lemma [20, $C[r, F]$ and $D[r, F]$ are not r.e.

Proof. Since this is a statement that does not involve enumerations of r.e. sets or partial recursive functions in any way, we are free to use such enumerations given by a Turing machine model. Recall the function $T$ of Lemma 17. Suppose $C[r, F]=$ $W_{e}$. Let $n$ be sufficiently large, and consider the longest closed interval $I(n)$ of integers starting with $\max \{\#(T(i)): i \leq n !)\} !$ !, that is contained in $W_{e}$, except possibly for the right endpoint, $x$. Here !! is the factorial of the factorial, which is used to avoid any issues surrounding ad hoc details of a Turing machine model, and \# is a standard indexing of the elements of IPOLY. So $x \geq \max \{\#(T(i)): i \leq$ $n$ !) $\}$ !!. Since $W_{e}$ is coinfinite, $I(n)$ exists, and $x \notin W_{e}$. Let $p$ be naturally chosen 
so that $W_{p}=I(n)$. Then clearly $p \leq n !$, and so $T(p)[-r, r] \subseteq[-x, x]$. But then $x \in W_{e}$, which is a contradiction.

The case of $D[r, F]$ is handled analogously as follows. Suppose $D[r, F]=W_{e}$. Let $n$ be sufficiently large, and consider the longest closed interval $I(n)$ of integers starting with $\max \{\#(T(i)): i \leq n !)\} !$ !, that is contained in $W_{e}$, except possibly for the right endpoint, $x$. Since $W_{e}$ is coinfinite, $I(n)$ exists, and $x \notin W_{e}$. Let $\left.I^{\prime}(n)=I(n) \cup[1, \max \{\#(T(i)): i \leq n !)\} ! !\right]$. Let $p$ be naturally chosen so that $W_{p}=I^{\prime}(n)$. Then clearly $p \leq n !$, and so $T(p)[-r, r] \subseteq[-x, x]$. But then $x \in W_{e}$, which is a contradiction.

Theorem 22. Let $r>2$ be a real number. Suppose $F: Z^{+} \rightarrow \Re^{+}$eventually dominates an unbounded recursive function from $Z^{+}$into $Z^{+}$, and for all sufficiently large $n, F(n) \leq(\log n)^{1 / 3}$. Then $C[r, F]$ and $D[r, F]$ meet every infinite r.e. set and are coinfinite. $C[r, F]$ and $D[r, F]$ are not r.e. and not co-r.e.

Proof. From Lemmas 20] and 21,

Lemma 23. Let $0<r<2$. There exists $c>0$ such that the following holds. For all sufficiently large $n$ there exists $P \in I P O L Y$ such that $P[-r, r] \subseteq[-c, c]$ and $\max (P Z)=n$.

Proof. See [3], Theorem 4.

Lemma 24. Let $0<r<2$. There exist real numbers $c_{1}>c_{2}>\ldots$ converging to 0 such that the following holds. For all sufficiently large $n$ there exists $P \in I P O L Y$ such that $P[-r, r] \subseteq\left[-c_{n}, c_{n}\right]$ and $\left|P Z \cap Z^{+}\right|=n$.

Proof. See [3], Theorem 7.

Theorem 25. Let $0<r<2$. Suppose $F: Z^{+} \rightarrow \Re^{+}$goes to infinity and $G$ : $Z^{+} \rightarrow \Re^{+}$is bounded below by a positive real number. Then $C[r, F]$ and $D[r, G]$ are cofinite.

Proof. Immediate from Lemmas 23 and 24 .

Corollary 26. If $0<r<2$, then $C[r]$ and $D[r]$ are recursive. If $r>2$, then $C[r]$ and $D[r]$ are nonrecursive. If $r>2$ is rational, then $C[r]$ and $D[r]$ are of Turing degree $\leq 0^{\prime}$.

Proof. Recall the definitions of $C[r]$ and $D[r]$ given just before Lemma 18 . The first claim follows from Theorem 25. The second claim follows from Theorem 22 The final claim is from Lemma 18.

In particular, note that

(1) $C[3 / 2]=\left\{n \in Z^{+}:(\exists P \in I P O L Y)(n=\max (P Z)\right.$ and $P[-3 / 2,3 / 2] \subseteq$ $\left.\left.\left[-(\log n)^{1 / 3},(\log n)^{1 / 3}\right]\right)\right\}$ is recursive;

(2) $C[3]=\left\{n \in Z^{+}:(\exists P \in I P O L Y)\left(n=\max (P Z)\right.\right.$ and $P[-3,3] \subseteq\left[-(\log n)^{1 / 3}\right.$, $\left.\left.\left.(\log n)^{1 / 3}\right]\right)\right\}$ is nonrecursive and $\leq 0^{\prime}$

(3) $D[3 / 2]=\left\{n \in Z^{+}:(\exists P \in I P O L Y)\left(n=\left|P Z \cap Z^{+}\right|\right.\right.$and $P[-3 / 2,3 / 2] \subseteq$ $\left.\left.\left[-(\log n)^{1 / 3},(\log n)^{1 / 3}\right]\right)\right\}$ is recursive;

(4) $D[3]=\left\{n \in Z^{+}:(\exists P \in I P O L Y)\left(n=\left|P Z \cap Z^{+}\right|\right.\right.$and $P[-3,3] \subseteq$ $\left.\left.\left[-(\log n)^{1 / 3},(\log n)^{1 / 3}\right]\right)\right\}$ is nonrecursive and $\leq 0^{\prime}$. 
It is not clear what happens if we use $[-2,2]$. Then is $C[2]$ recursive? Is $D[2]$ recursive?

In general we can ask for good necessary and/or sufficient conditions on $r, F$ such that $C[r, F]$ is recursive, or $D[r, F]$ is recursive. This may involve a rather delicate interaction between approximation theory, recursion theory, and number theory (aspects of the latter involved in the solution to Hilbert's 10th problem).

\section{NATURAL NONRECURSIVE RELATIONS}

We present some natural binary and ternary relations on $Z^{+}$whose nonrecursiveness is an immediate consequence of Corollary 26.

Theorem 27. The following binary relations on $Z^{+}$are not r.e., not co-r.e., and $\leq 0^{\prime}$.

(1) $\left\{(n, m) \in Z^{+} \times Z^{+}:(\exists P \in I P O L Y)(n=\max (P Z)\right.$ and $P[-3,3] \subseteq$ $[-m, m])\}$

(2) $\left\{(n, m) \in Z^{+} \times Z^{+}:(\exists P \in I P O L Y)\left(n=\left|P Z \cap Z^{+}\right|\right.\right.$and $P[-3,3] \subseteq$ $[-m, m])\}$.

Proof. Let $F: Z^{+} \rightarrow R^{+}$be given by $F(n)=\left\lfloor(\log n)^{1 / 3}\right\rfloor$ and $r=3$. By Theorem 222 $C[r, F]$ and $D[r, F]$ are not r.e. or co-r.e. Now $n \in C[r, F]$ if and only if $(n, F(n))$ lies in the first set, and $n \in D[r, F]$ if and only if $(n, F(n))$ lies in the second set. Hence the first and second sets cannot be r.e. or co-r.e.

Theorem 28. The following ternary relations on $Z^{+}$are not r.e., not co-r.e., and $\leq 0^{\prime}$.

(1) $\left\{(n, m, r) \in Z^{+} \times Z^{+} \times Z^{+}:(\exists P \in I P O L Y)(n=\max (P Z)\right.$ and $P[-m, m]$ $\subseteq[-r, r])\}$

(2) $\left\{(n, m, r) \in Z^{+} \times Z^{+} \times Z^{+}:(\exists P \in I P O L Y)\left(n=\left|P Z \cap Z^{+}\right|\right.\right.$and $P[-m, m]$ $\subseteq[-r, r])\}$.

Proof. The obvious reductions of the two binary relations in Theorem 27 to these ternary relations are such that if the latter are r.e. (co-r.e.) so are the former.

We now present a 16-ary relation $R$ on the extended integers $Z^{e}=Z \cup\{-\infty, \infty\}$ that is particularly natural. For $a, b \in Z^{e}$, we let $(a, \ldots, b)=\{i \in Z: a<i<b\}$ and $(a, b)=\{x \in \Re: a<x<b\}$.

$R\left(n_{1}, \ldots, n_{16}\right)$ if and only if there exists $P \in I P O L Y$ such that

(1) $P\left(n_{1}, \ldots, n_{2}\right) \subseteq\left(n_{3}, \ldots, n_{4}\right)$;

(2) $P\left(n_{5}, n_{6}\right) \subseteq\left(n_{7}, n_{8}\right)$;

(3) $P\left(n_{9}, \ldots, n_{10}\right) \nsubseteq\left(n_{11}, \ldots, n_{12}\right)$; and

(4) $P\left(n_{13}, n_{14}\right) \nsubseteq\left(n_{15}, n_{16}\right)$.

The use of open intervals here causes a technical problem which is easily resolved. Define $C(r, F)$ and $D(r, F)$ to be the same as $C[r, F]$ and $D[r, F]$ except that $[-r, r]$ is replaced by $(-r, r)$ and $[-F(n), F(n)]$ is replaced by $(-F(n), F(n))$.

Lemma 29. Let $r>2$ be a real number. Suppose $F: Z^{+} \rightarrow \Re^{+}$eventually dominates an unbounded recursive function from $Z^{+}$into $Z^{+}$, and for all sufficiently large $n, F(n) \leq(\log n)^{1 / 3}$. Then $C(r, F)$ and $D(r, F)$ meet every infinite r.e. set and are coinfinite. $C(r, F)$ and $D(r, F)$ are not r.e. and not co-r.e. 
Proof. Note that $C[r+1, F / 2] \subseteq C(r, F) \subseteq C[r, F]$. By Theorem [22] $C[r+1, F / 2]$ meets every infinite r.e. set, and $C[r, F]$ is coinfinite. Hence $C(r, F)$ meets every infinite r.e. set and is coinfinite. The proof of Lemma 21 also works for $C(r, F)$. $D(r, F)$ is treated in the same way.

Theorem 30. The 16-ary relation $R$ on $Z^{e}$ is not r.e. and not co-r.e.

Proof. Let $F: Z^{+} \rightarrow \Re^{+}$be given by $F(n)=\left\lfloor(\log n)^{1 / 3}\right\rfloor$ and $r=3$. By Theorem [29, $C(r, F)$ is not r.e. or co-r.e. Note that $n \in C(r, F)$ if and only if $R(-\infty, \infty,-\infty, n+1,-r, r,-F(n), F(n),-\infty, \infty,-\infty, n,-\infty, \infty, 0,0)$.

Note. Recent recursion theoretic work of Davie and Solovay shows that the set $A$ of section 2 is Turing complete (of Turing degree $0^{\prime}$ ). It is expected to show that $B$ is Turing complete, as well as the sets at the end of section 4 . A consequence would be that the binary and ternary relations in this section are also Turing complete (of Turing degree $0^{\prime}$ ), and that $R \geq 0^{\prime}$. Is $R$ Turing complete?

We wish to thank the referee for helpful suggestions. In particular, the referee has pointed out that in [4, a sharper version of the trick used in the proof of Lemma 17 can be found, which, in our notation, shows that we can require $T(e)=$ $W_{e} \cup Z^{-}$. The referee suggests the possibility of using this to obtain additional natural nonrecursive sets by using $P Z=\{x \in Z: x \leq n\}$ as an alternative to our $n=\max (P Z)$ and $n=\left|P Z \cap Z^{+}\right|$. This suggestion will be pursued elsewhere.

\section{REFERENCES}

[1] P. Odifreddi, Classical Recursion Theory, Studies in Logic and the Foundations of Mathematics, volume 125, North-Holland, 1989. MR.90d:03072

[2] M. Davis, Unsolvable problems, in Jon Barwise, editor, Handbook of Mathematical Logic, volume 90 of Studies in Logic and the Foundations of Mathematics, Chapter C.2, pages 567594, North Holland, 1977. MR56:15351

[3] T. Erdélyi, H. Friedman, The number of certain integral polynomials and nonrecursive sets of integers, Part 1, this issue.

[4] H. Putnam, An unsolvable problem in number theory, Journal of Symbolic Logic, Vol. 25, No. 3, Sept. 1960, 220-232. MR.28:2048

Department of Mathematics, Ohio State University, Columbus, Ohio 43210

E-mail address: friedman@math.ohio-state.edu 